\title{
Protective effect of diltiazem on myocardial ischemic rats induced by isoproterenol
}

\author{
YUEJIAO WEI $^{1,2}$, TIANYU MENG ${ }^{1,2}$ and CHAOFENG SUN ${ }^{1,2}$ \\ ${ }^{1}$ Department of Cardiovascular Medicine, The First Affiliated Hospital of Xi'an Jiaotong University; \\ ${ }^{2}$ Shaanxi Key Laboratory of Molecular Cardiology, Xi'an Jiaotong University, Xi'an, Shaanxi 710061, P.R. China
}

Received March 1, 2017; Accepted August 31, 2017

DOI: $10.3892 / \mathrm{mmr} .2017 .7906$

\begin{abstract}
The aim of the present study was to analyze the effect of diltiazem on myocardial fibrosis and remodeling of connexin43 (Cx43) in myocardial ischemic rats and mechanisms underlying these processes. A total of 36 Sprague-Dawley rats were randomly allocated into three groups (control, isoproterenol and isoproterenol with diltiazem). The myocardial ischemic model was established by $5 \mathrm{mg} / \mathrm{kg} / \mathrm{day}$ isoproterenol administration for 7 days, and the diltiazem group received $25 \mathrm{mg} / \mathrm{kg} /$ day diltiazem for 4 weeks. Following the treatment, paraffin sections were prepared to observe microstructural changes and to evaluate the concentration of $\mathrm{Ca}^{2+}$ in myocardium. The expression of transforming growth factors- $\beta 1$ (TGF- $\beta 1$ ), mothers against decapentaplegic homologues (Smad)2 and 7 and $\mathrm{Cx} 43$, were analyzed by reverse transcription-quantitative polymerase chain reaction and western blotting. The percentage Cx43 expression in intercalated disks was evaluated using immunohistochemistry. Fibrosis did not differ significantly between the control and the diltiazem-treated group. The concentration of $\mathrm{Ca}^{2+}$ increased in the myocardium of model rats. The expression of Smad7 and $\mathrm{Cx} 43$ was decreased in the rat model, while the expression of TGF- $\beta 1$ and Smad 2 was increased. There was a significant decrease in the relative abundance of intercalated disk Cx43 in the model group. The results of the present study suggest that diltiazem may serve a protective role during remodeling of myocardial ischemia, especially in fibrosis and Cx43 remodeling.
\end{abstract}

\section{Introduction}

Myocardial ischemia has become a common life-threatening disease causing many adverse effects on the heart, including a decrease in myocardial cell aerobic metabolism and a

Correspondence to: Professor Chaofeng Sun, Department of Cardiovascular Medicine, The First Affiliated Hospital of Xi'an Jiaotong University, 277 West Yanta Road, Xi'an, Shaanxi 710061, P.R. China

E-mail: csun1@163.com

Key words: diltiazem, myocardial ischemia, fibrosis, connexins reduction in the capacity to maintain the heart activity during periods of low oxygen supply (1). Myocardial ischemia can initially present no symptoms but subsequently lead to serious clinical consequences. Although reperfusion therapy can rescue myocardial ischemia and myocardial stunning, damaged myocardia and newly forming myocardial injuries caused by reoxygenation still induce heart failure and ventricular remodeling even in patients who received reperfusion therapy $(2,3)$. Ventricular remodeling following myocardial ischemia is an important factor contributing to chronic heart failure. Myocardial fibrosis and connexin43 (Cx43) remodeling are important forms of ventricular remodeling which affect myocardial systolic and diastolic function and lead to cardiac arrhythmia, increasing the incidence of sudden death $(4,5)$.

Myocardial fibrosis is characterized by an excessive deposition of extracellular matrix proteins by cardiac fibroblasts (CFs) in the myocardial tissue and imbalanced abundance of different types of collagen, which reduces tissue compliance and limits the diastolic filling pressure $(6,7)$. Fibrosis of coronary arteries thickens the wall of the heart, causes luminal narrowing and decreases myocardial elasticity, reducing the blood supply for myocardial cells (8). The majority of causes of heart disease involve pathological myocardial fibrosis. Myocardial fibrosis is a complex pathological process in which transforming growth factors- $\beta 1$ (TGF- $\beta 1$ ) is the most important growth factor. TGF- $\beta 1$ can promote differentiation of CFs, activation of the renin-angiotensin-aldosterone system and cause increased abundance of niacinamide adenine dinucleotide phosphate (NADPH). All of the above are mechanisms of fibrosis $(9,10)$. Mothers against decapentaplegic homologue (Smad) proteins are the main effector molecules in the TGF- $\beta 1$ signaling pathway, which regulates the expression of downstream genes and promotes fibrosis (11). The Smad protein family can be divided into three groups: i) Receptor regulated smads (R-Smads) including Smad1, 2, 3, 5 and 8; ii) common partner smads (Co-Smads) including Smad4; and iii) inhibitory smads (I-Smads) including Smad6 and 7. I-Smads can compete with R-Smads for binding with their receptor. Therefore, I-Smads can prevent phosphorylation of R-Smads and inhibit TGF- $\beta 1$ signaling pathway. Therefore, the expression of TGF- $\beta 1$, Smad 2 and 7 serve a role in myocardial fibrosis.

Connexins are transmembrane ion-channel proteins that form gap junctions between adjacent cardiomyocytes and transfer ions or molecules between cells, coupling them 
electrically (12). Cx43 is the predominant isoform in the heart, mainly present in ventricular tissues. Changes in Cx43 expression and distribution are closely associated with electrical remodeling of ventricular tissues (13).

In the treatment of myocardial ischemia, reperfusion is not the main goal and preventing harmful changes in the myocardial structure is equally important. Calcium channel blockers $(\mathrm{CCBs})$ are common cardiovascular medicines that have been effectively used in the treatment of arrhythmia, myocardial ischemia and hypertension. Although CCBs have been reported to alleviate myocardial remodeling, previous studies have only been limited to studying myocardial cells, including a preliminary study on the quality index of the heart (14-16). The underlying molecular mechanisms of structural and electrical remodeling, have yet to be elucidated. In the present study, the effects of diltiazem on isoproterenol-induced myocardial fibrosis and $\mathrm{C} \times 43$ expression have been investigated.

\section{Materials and methods}

Materials. Male 8-week-old Sprague-Dawley rats (body weight, 200-250 g; n=36) were obtained from Xi'an Jiaotong University (Xi'an, China). Rats were reared at a constant-temperature of $22-25^{\circ} \mathrm{C}$ with a 12 -h light/dark cycle, and given a standard diet and unlimited access to drinking water. The present study was approved by the Institutional Laboratory Animal Care and Use Committee of the School of Xi'an Jiaotong University (approval no. 2016-160). Isoproterenol was purchased from Sigma-Aldrich (Merck KGaA, Damstadt, Germany). Diltiazem was purchased from Simcare Drug Store (Nanjing, China). Other reagents were of commercial analytical grade.

Experimental protocol. Following acclimatization for 1 week, experimental rats $(\mathrm{n}=36)$ were randomly divided into three groups, 12 rats each: i) Control (CTL); ii) isoproterenol (ISO); and iii) isoproterenol with diltiazem (ISO_DIL). Myocardial ischemia in rats (ISO and ISO_DIL) was induced by subcutaneous isoproterenol injection (isoproterenol was dissolved in physiological saline and administered at $5 \mathrm{mg} / \mathrm{kg}$ body weight/day) at 24-h intervals for 7 days, as previously described (17). The CTL group was injected with equal volumes of physiological saline at the same time intervals. Following the second isoproterenol injection, the ISO_DIL group was given $25 \mathrm{mg} / \mathrm{kg} /$ day diltiazem for 4 weeks. The CTL and ISO group were given $1 \mathrm{ml} /$ day physiological saline for 4 weeks. The treatments involved oral administration. At $12 \mathrm{~h}$ following administration of the final dose of diltiazem, cervical dislocation was performed and a piece of tissue from the apex of the left ventricle was excised immediately form each rat, washed with chilled isotonic saline and used for analysis.

Determination of heart weight index (HWI). Whole hearts were excised (excluding large blood vessels and connective tissue) wiped with filter paper and weighed. HWI was calculated as heart weight/body weight.

Histopathological analysis. Hearts were fixed in $10 \%$ formalin solution and embedded in paraffin wax at $4^{\circ} \mathrm{C}$ for $24 \mathrm{~h}$. Transverse sections, 4-mm thick, were cut and stained as previously described (18). Hematoxylin and eosin (H\&E) and
Masson stains were used to observe microstructural changes and detect the quantities of collagen (magnification, $\mathrm{x} 400$ ). Briefly, hematoxylin dye was applied for $5 \mathrm{~min}$, the acid dip for $5 \mathrm{sec}$ and ammonia water for another $5 \mathrm{sec}$. Subsequently, sections were washed with water for $1 \mathrm{~h}$ followed by washes with distilled water for another $5 \mathrm{~min} .70$ and $90 \%$ alcohol dehydration step followed for $10 \mathrm{~min}$. Finally, sections were put into alcohol eosin staining for 2-3 min. For the Masson stain, sections were washes with tap water and distilled water for $2 \mathrm{~min}$, followed by Harris's wood dye for 1-2 min. rinses with water followed for $30 \mathrm{~min}$. All incubations occurred at room temperature. Additionally, Masson acid dye was applied for 5-10 min, 1\% phosphomolybdic acid aqueous solution for another 3-5 $\mathrm{min}$ and $1 \%$ aniline blue dye for $5 \mathrm{~min}$. Finally, $1 \%$ glacial acetic acid aqueous solution was added for $5 \mathrm{sec}$. All incubations occurred at room temperature and the visualization occurred using a bright field Olympus+CX23 microscope (Olympus Corporation, Tokyo, Japan).

The degree of cardiac fibrosis was determined based on the area of fibrosis/total tissue area (\%), as previously described (18).

Concentration of $\mathrm{Ca}^{2+}$. Myocardial tissue was homogenized by heat-treatment for $6 \mathrm{~h}$ at $85^{\circ} \mathrm{C}$. Centrifugation $\left(7,500 \mathrm{xg}\right.$ at $4^{\circ} \mathrm{C}$ for $15 \mathrm{~min}$ ) separated the supernatant containing $\mathrm{Ca}^{2+}$ from the pellet. Methyl phenol blue thyme in alkaline solution was added to the supernatant and formed blue-colored complexes with $\mathrm{Ca}^{2+}$. The concentration of $\mathrm{Ca}^{2+}$ in the supernatant was evaluated by color comparison with a standard solution, as previously described (19).

Western blotting. Western blotting analyses were performed according to a previously described method (20). Frozen heart tissue was homogenized in lysis buffer. Following two centrifugation extractions at $4^{\circ} \mathrm{C}, 12,000 \mathrm{x}$ g for $15 \mathrm{~min}$ and at $4^{\circ} \mathrm{C}$, $12,000 \mathrm{x} \mathrm{g}$ for $10 \mathrm{~min}$, the resulting supernatant was used for analysis. Protein content was determined using the Bradford protein assay. Diluted tissues (20 $\mu \mathrm{g}$ protein/lane) were separated by electrophoresis in 10\% polyacrylamide gels and the separated proteins were transferred onto polyvinylidene fluoride membranes for visualization. Nonspecific binding sites were blocked with 5\% skimmed milk at room temperature for $30 \mathrm{~min}$. Following washing with $0.05 \%$ Tris-buffered saline (TBST) with $0.05 \%$ Tween), membranes were incubated overnight at $4^{\circ} \mathrm{C}$ with primary monoclonal antibodies specific for TGF- $\beta 1$ (1:800, cat. no. sc-146; Santa Cruz Biotechnology, Inc., Dallas, TX, USA), Smads (1:800, Smad2; cat. no. SAB4300251 and Smad7, cat. no. SAB4200345; both from Sigma-Aldrich; Merck KGaA, Darmstadt, Germany, Cx43 (1:800, cat. no. 3512; Cell Signaling Technology, Inc., Dancers, MA, USA) and GAPDH (1:1,000, cat. no. SAB2100894; Sigma-Aldrich; Merck KGaA) and then with Goat anti rabbit IgG $(1 ; 5,000$, cat. no. 1706515; Bio-Rad Laboratories, Inc., Hercules, CA, USA) at room temperature for $2 \mathrm{~h}$. Following washing with TBS with Tween-20, proteins were visualized using enhanced chemiluminescence substrate and a ChemiDoc XRS Imaging system (Bio-Rad Laboratories, Inc.). GAPDH was used as a loading control.

Reverse transcription-quantitative polymerase chain reaction $(R T-q P C R)$. Following removal and washing with cold saline, 
Table I. Primer sequences used for reverse transcription-quantitative polymerase chain reaction.

\begin{tabular}{lllr}
\hline Gene/site & \multicolumn{1}{c}{ Forward primer (5'-3') } & Reverse primer (5'-3') & Product length (bp) \\
\hline Cx43 & GCCGCAATTACAACAAGCAA & TTGGCATTCTGGTTGTCGTC & 142 \\
TGF- 31 & GAGAAGCGGTACCTGAACCC & GGCGAAAGCCCTCAATTTCC & 234 \\
Smad2 & CCATCTTGCCATYCACTCCG & AAGCTCATCTAATCGTCCTG & 176 \\
Smad7 & CCATCACCTTAGCCGACTCTG & AAATCCATCGGGTATCTGGAGTA & 69 \\
GAPDH & GGAAAGCTGTGGCGTGAT & AAGGTGGAAGAATGGGAGTT & 308 \\
\hline
\end{tabular}

Cx43, connexin43; Smad, mothers against decapentaplegic homologues; TGF- $\beta 1$, transforming growth factors- $\beta 1$.

hearts were frozen in liquid nitrogen and preserved at $-80^{\circ} \mathrm{C}$. RT-qPCR was performed as previously described (21). Total RNA was extracted from myocardial tissue using TRIzol reagent (Invitrogen; Thermo Fisher Scientific, Inc., Waltham, MA, USA). RNA was then reverse transcribed by RevertAid First-Strand cDNA Synthesis kit (Thermo Fisher Scientific, Inc.). The mRNA expression level of Cx43 was measured by RT-qPCR. The expression of the transcript was normalized to the mean GAPDH mRNA expression (22) and determined using a 7300 Real-Time PCR System SDS software version 1.4 (Applied Biosystems; Thermo Fisher Scientific, Inc.). The primer sequences are summarized in Table I.

Immunofluorescence assay. As previously described (23), fixed hearts were coated with optimal cutting temperature compound (Sakura Finetek USA, Inc., Torrance, CA, USA) and cut into $6 \mu \mathrm{m}$-thick sections. Sections of left ventricular tissue were incubated with the primary antibodies for Cx43 (1:100; cat. no. 3512; Cell Signaling Technology, Inc.) overnight at $4^{\circ} \mathrm{C}$. Following three washes with $0.1 \mathrm{~mol} / 1$ phosphate-buffered saline (PBS), 5 min each, the sections were incubated with Fluorescein-Conjugated AffiniPure Goat Anti-Rabbit IgG(H+L) (1:50 dilution, cat. no. ZF-0311; ZSGB-BIO; OriGene Technologies, Inc., Rockville, MD, USA) at $37^{\circ} \mathrm{C}$ for $1 \mathrm{~h}$ in the dark. Then, following rinsing with PBS three times, the images of the sections were captured using a laser confocal microscope (Olympus Soft Imaging Solutions, Münster, Germany). The absorbance was measured at a wavelength of $488 \mathrm{~nm}$ and analyzed using Image Pro-Plus software (version 7.0; Media Cybernetics, Inc., Rockville, MD, USA).

Statistical analysis. The experimental data were analyzed by one-way analysis of variance, followed by the Fisher's least significant difference test. using SPSS software (version 10.0; SPSS, Inc., Chicago, IL, USA) and are expressed as the mean \pm standard deviation. $\mathrm{P}<0.05$ was considered to indicate a statistically significant difference.

\section{Results}

$H W I$. HWIs were significantly greater in ISO rats compared with the CTL group and ISO_DIL groups $(\mathrm{P}<0.05$; Table II).

Ventricular histological observation. To assess the differences in structural remodeling between subgroups, ventricular
Table II. Effect of diltiazem on HWI.

\begin{tabular}{ll}
\hline Group & HWI (mg/g) \\
\hline Control & $3.106 \pm 0.809$ \\
Model & $3.916 \pm 0.235^{\text {a }}$ \\
Diltiazem & $3.341 \pm 0.575^{\mathrm{b}}$
\end{tabular}

Data are presented as the mean \pm standard deviation ( $n=12 /$ group). ${ }^{\mathrm{a}} \mathrm{P}<0.05$ vs. the control group. ${ }^{\mathrm{b}} \mathrm{P}<0.05$ vs. the untreated model group. HWI, heart weight index.

Table III. Concentration of $\mathrm{Ca}^{2+}$.

\begin{tabular}{ll}
\hline Group & $\mathrm{Ca}^{2+}(\mathrm{nmol} / \mathrm{l})$ \\
\hline Control & $161.976 \pm 7.476$ \\
Model & $429.973 \pm 23.592^{\mathrm{a}}$ \\
Diltiazem & $253.636 \pm 25.250^{\mathrm{b}}$
\end{tabular}

Data are presented as the mean \pm standard deviation ( $\mathrm{n}=12$ /group). ${ }^{\mathrm{a}} \mathrm{P}<0.01$ vs. the control group. ${ }^{\mathrm{b}} \mathrm{P}<0.01$ vs. the untreated model group.

histology was analyzed. Light microscopy of H\&E- and Masson-stained tissue sections from CTL and ISO_DIL rats demonstrated myocardium with normal cellular structure and continuous myofibril structure with adjacent myofibrils. However, tissue sections in ISO rats exhibited obvious myocardial cell swelling, degeneration and hyperplasia of large connective tissues. The percentage of fibrotic area increased significantly in the ISO group compared with the CTL group, and decreased significantly in the ISO_DIL group compared with the ISO model group (both $\mathrm{P}<0.001$; Fig. 1).

Concentration of $\mathrm{Ca}^{2+}$. Compared with CTL rats, concentration of $\mathrm{Ca}^{2+}$ in myocardium increased significantly in ISO rats. It has been demonstrated that high calcium concentrations contribute to the pathogenesis of myocardial ischemia injury (24). Concentration of $\mathrm{Ca}^{2+}$ significantly decreased the ISO_DIL group $(\mathrm{P}<0.05$; Table III).

Changes in the TGF- $\beta 1 /$ Smads signaling pathway. Both protein (Fig. 2) and mRNA (Fig. 3) expression of TGF- $\beta 1$ and Smad2 decreased in ISO_DIL rats compared with the ISO 


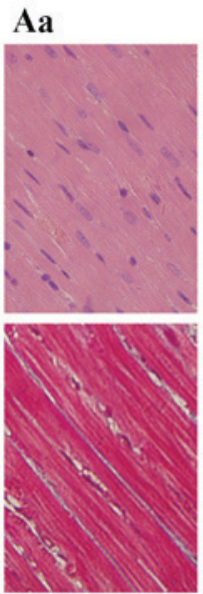

Control
Ab
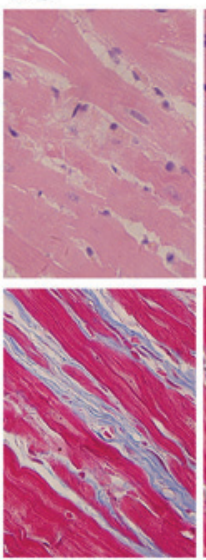

Model
Ac

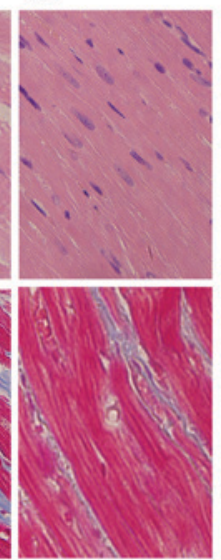

Diltiazem
B

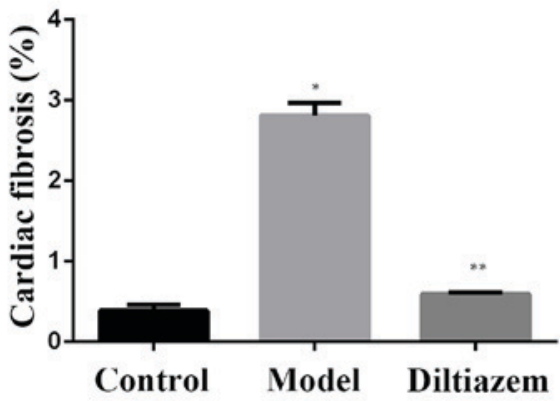

Figure 1. Histopathological analysis of rat myocardium. (Aa) Myocardial tissue and myofibril structure in control rats. (Ab) Myocardial ischemia induced by isoproterenol demonstrating swelling and irregular arrangement of cells, connective tissue hyperplasia was also visible. (Ac) Myocardium without any cell swelling and degeneration in rats treated with diltiazem. Top three panels, ventricular hematoxylin and eosin stain (magnification, $\mathrm{x} 400$ ) and bottom three panels, ventricular Masson stain (magnification, $\mathrm{x} 400$ ). (B) Cardiac fibrosis (\%) in different groups. Data are presented as the mean \pm standard deviation. ${ }^{*} \mathrm{P}<0.001$ vs. control group; ${ }^{* *} \mathrm{P}<0.001$ vs. untreated model group.

A

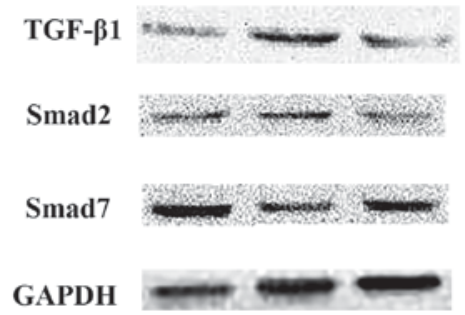

B a

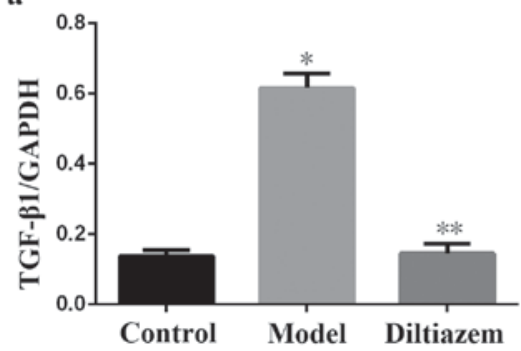

B b

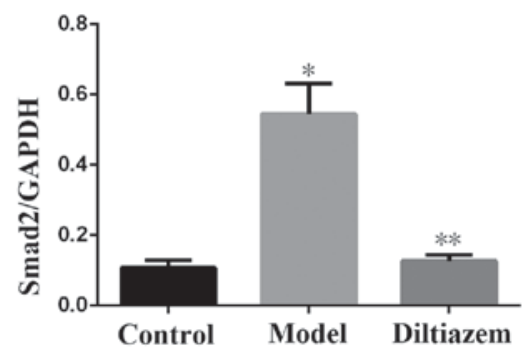

B c

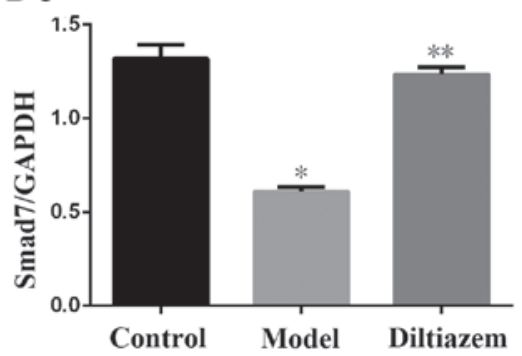

Figure 2. Expression of the TGF- $\beta 1 /$ Smads signaling pathway. (A) Western blotting of TGF- $\beta 1$ and Smad 2 and 7 from the rat left ventricle in the control, model and diltiazem groups, from left to right respectively. (Ba) TGF- $\beta 1$, (Bb) Smad2, (Bc) Smad7 protein expression level relative to the marker (GAPDH). Data are presented as the mean \pm standard deviation. ${ }^{*} \mathrm{P}<0.001$ vs. the control group. ${ }^{* *} \mathrm{P}<0.001$ vs. the untreated model group. TGF- $\beta 1$, transforming growth factor- $\beta 1$; Smad, mothers against decapentaplegic homologues.

group, while the expression of Smad7 increased in ISO_DIL group compared with the ISO group (all $\mathrm{P}<0.001)$.

Remodeling of Cx43. As presented in Fig. $4 \mathrm{~A}$ and $\mathrm{B}$, the protein expression of $\mathrm{Cx} 43$ was decreased in the model group compared with the ISO_DIL and CTL groupa. However, the expression of Cx43 mRNA did not differ significantly between all three groups (Fig. 4C). This observation suggests that diltiazem can improve the remodeling of connexins in a quantitative manner following myocardial ischemia, but the mechanism maybe not involve control at the mRNA level. Localization of $\mathrm{Cx} 43$ was determined using immunohistochemistry and confocal imaging. Only longitudinal sections of myocytes were evaluated. Intercalated disks of all myocytes were identified in high-power field, and image quantification software was used to quantify the signal intensity and to calculate the ratio of intercalated disk connexins to the total intensity (IC-disk $\%=$ IC-disk intensity/total intensity x100\%). In the ISO_DIL group, the percentage of $\mathrm{Cx} 43$ at intercalated disks did not change compared with the CTL group. However, in the ISO group, the relative percentage of $\mathrm{Cx} 43$ at intercalated disks decreased significantly compared with the CTL and ISO_DIL groups (both $\mathrm{P}<0.001$; Fig. 5). 
A

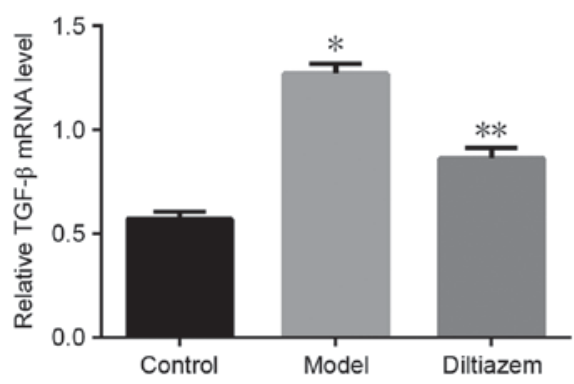

C

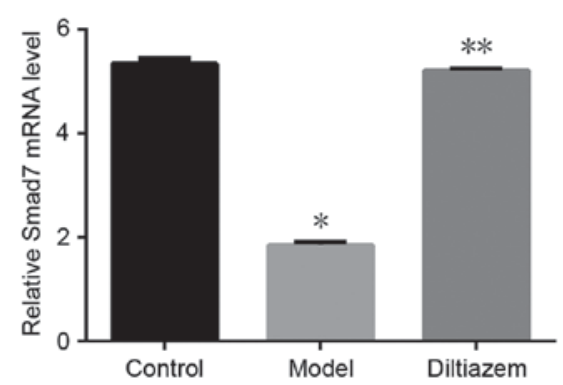

B

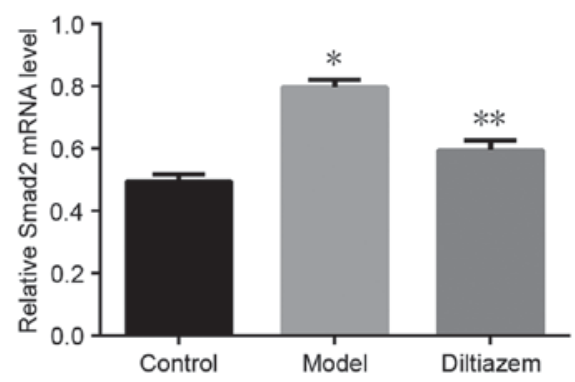

Figure 3. mRNA expression of the TGF- $\beta 1 /$ Smads signaling pathway. mRNA levels of (A) TGF- $\beta 1$ (B) Smad2 and (C) Smad7 were normalized to GAPDH. All data were expressed as the mean \pm standard deviation. ${ }^{*} \mathrm{P}<0.001$ vs. the control group; ${ }^{* *} \mathrm{P}<0.001$ vs. the untreated model group. TGF- $\beta 1$, transforming growth factors- $\beta 1$; Smad, mothers against decapentaplegic homologues.

A

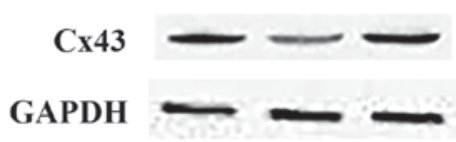

B

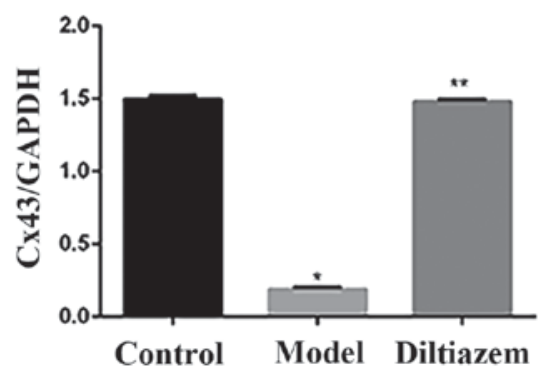

C

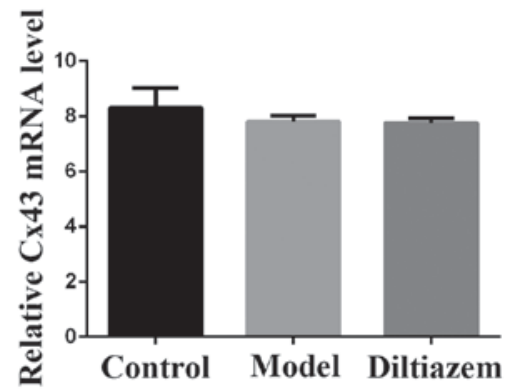

Figure 4. Cx43 expression. (A) Representative western blot images of Cx43 from the rat left ventricle in the control, model and diltiazem groups, from left to right, respectively. (B) Cx43 and (C) mRNA expression levels, normalized to GAPDH. Data are expressed as the mean \pm standard deviation. "P<0.001 vs. control group; ${ }^{* *} \mathrm{P}<0.001$ vs. untreated model group. Cx43, connexin 43 .

Aa

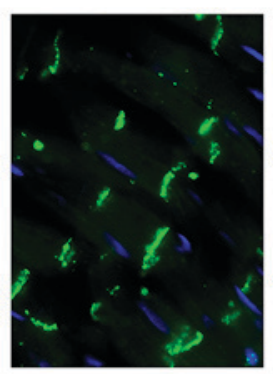

b

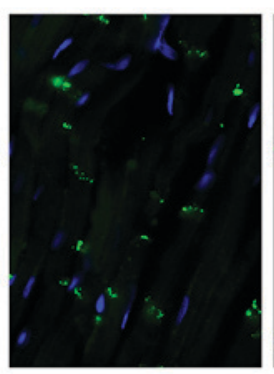

c

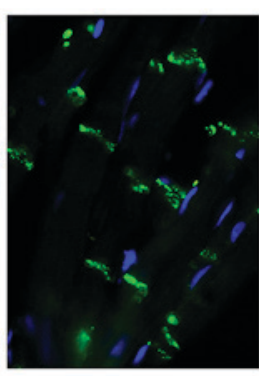

B

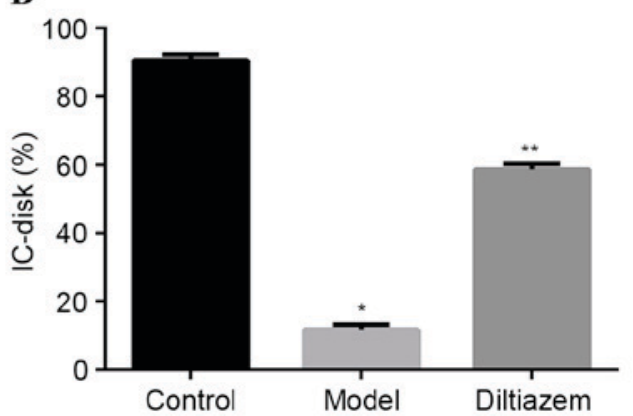

Figure 5. Change in the Cx43 distribution in each group. (Aa) Majority of Cx43 were distributed in intercalated disks in the control group. (Ab) Irregular distribution of $\mathrm{Cx} 43$ in the model group, predominantly located on flanking cells. (Ac) In the diltiazem group, some Cx43 transferred from the intercalated disk to the lateral side of cells. (B) IC-disk (\%) in the control, model and diltiazem group. ${ }^{*} \mathrm{P}<0.001$ vs. control the group; ${ }^{* *} \mathrm{P}<0.001$ vs. the untreated model group. Cx43, connexin43; IC-disk, intercalated disk. 


\section{Discussion}

In the present study, the cardioprotective effects of diltiazem in myocardial ischemic rats induced by isoproterenol were evaluated. By studying the histopathological changes, translation and expression of the associated proteins, an attempt was made to determine possible mechanisms underlying the therapeutic efficiency of diltiazem. The results of the present study demonstrated that diltiazem served a protective role during myocardial ischemia, especially in fibrosis and $\mathrm{Cx} 43$ remodeling.

Isoproterenol, a known b-adrenoceptor agonist, induces myocardial ischemia by increasing the force and frequency of myocardial contractions $(18,25-27)$ and the concentration of intracellular $\mathrm{Ca}^{2+}(28) . \mathrm{Ca}^{2+}$ serves an important role in maintaining myocardial systolic and diastolic functions, and normal myocardial rhythmicity and excitability. As a secondary intracellular messenger, $\mathrm{Ca}^{2+}$ participates in a variety of pathophysiological processes and signal transduction pathways (29).

During ischemia, anaerobic myocardial metabolism predominates, reducing the amount of ATP produced. As a result, the activity of sodium-potassium $\left(\mathrm{Na}^{+}-\mathrm{K}^{+}\right)$pumps on cell membranes, which requires energy from ATP, gradually declines. Therefore, the material exchange dependent on the $\mathrm{Na}^{+}-\mathrm{K}^{+}$electrochemical potential energy is distorted, leading to an increase in the intracellular $\mathrm{Na}^{+}$concentration. The aforementioned changes lead to an increase in anaerobic glycolysis and accumulation of lactic acid, which in turn causes intracellular acidification, increased $\mathrm{Na}^{+}-\mathrm{H}^{+}$exchange and increased intracellular $\mathrm{Na}^{+}$concentration. Accumulation of intracellular $\mathrm{Na}^{+}$activates $\mathrm{Na}^{+}-\mathrm{Ca}^{2+}$ exchanging proteins located on the plasma membrane and accelerates $\mathrm{Ca}^{2+}$ transport into the cytoplasm, increasing intracellular $\mathrm{Ca}^{2+}$ concentration. In the present study, $\mathrm{Ca}^{2+}$ concentration in myocardium increased significantly in ISO rats compared with the CTL group. Diltiazem effectively decreased the $\mathrm{Ca}^{2+}$ concentration.

Accumulation of intracellular $\mathrm{Ca}^{2+}$, which promotes mitosis, can induce cell proliferation, especially of myocardial fibroblasts. The results of the present study were consistent with those of previous studies (30-32). Compared with the CTL and ISO_DIL groups, HWI in the ISO rats was greater and the morphology of ventricular tissue was more abnormal, demonstrating cardiomyocyte hypertrophy, disordered tissue arrangement and connective tissue hyperplasia. These results confirmed the possibility of myocardial reconstruction following ischemia. At present, mechanisms underlying $\mathrm{Ca}^{2+}$-induced proliferation of fibroblasts remain to be elucidated. According to one hypothesis, secondary intracellular messenger $\mathrm{Ca}^{2+}$ affects the formation of fibroblasts and promotes their proliferation (33). By participating in signal transduction pathways of some cell growth factors, $\mathrm{Ca}^{2+}$ can also promote myocardial fibrosis. The present study demonstrated that the expression of TGF- $\beta 1$ and Smad2 was decreased in ISO rats, while Smad7 was upregulated. These results demonstrated similar patterns in protein and mRNA levels with previous studies. Smad7 is an endogenous inhibitory factor of TGF- $\beta 1$. Following myocardial ischemia, expression of Smad7 is reduced, which can lead to the excessive activation of TGF- $\beta 1$ (34). Previous research demonstrated that $\mathrm{Ca}^{2+}$ can interact with angiotensin II and aldosterone to stimulate fibroblast proliferation and collagen synthesis. Angiotensin II-aldosterone-stimulated myocardial fibrosis in rats is reduced dramatically by CCBs (35). The present study demonstrated that diltiazem serves a protective role in myocardial fibrosis but further studies are required to investigate the underlying mechanisms.

Remodeling of $\mathrm{Cx} 43$, the major connexin isoform in the ventricle, is one of the most harmful consequences of myocardial ischemia. Decreased Cx43 expression, its heterogeneity and lateralization of connexin distribution are considered forms of $\mathrm{Cx} 43$ remodeling. According to the present study, the expression of $\mathrm{Cx} 43$ decreased markedly in the ISO group compared with CTL and ISO_DIL groups. Percentage Cx43 expression localized to intercalated disks was evaluated using immunohistochemistry and confocal imaging. Relative abundance of $\mathrm{Cx} 43$ at intercalated disks did not differ significantly between CTL and ISO_DIL groups. However, there was a significant decrease in the ISO group compared with both CTL and ISO_DIL groups. Cx43 distribution, particularly its redistribution from cell-cell gap junctions to lateral margins, was investigated in the present study. Given the implication of connexin in cell-to-cell coupling, changes in connexin expression and distribution were expected to have an influence on cardiac conduction (36). Decreased Cx43 expression and heterogeneity could alter the isotonicity of electric conduction and lead to reduction of the rate of electrical conduction and reentry. Lateralization of $\mathrm{Cx} 43$ can lead to delayed afterdepolarization $(37,38)$. The aforementioned remodeling of Cx43 in pathological conditions can lead to arrhythmia.

Previous studies confirmed that intracellular $\mathrm{Ca}^{2+}$ concentration regulates the permeability of gap junctions composed of Cx43 (39,40). Increased abundance of intracellular $\mathrm{Ca}^{2+}$ can activate calcium-dependent proteolytic enzymes (calpains), which can degrade a variety of proteins, and change cell structure and function (41). Therefore, activation of calpains caused by the accumulation of intracellular $\mathrm{Ca}^{2+}$ may be one of the most important mechanisms in connexin remodeling following myocardial ischemia. In the present study, the expression of Cx43 mRNA did not change between the three groups of interest, whereas the expression of $\mathrm{Cx} 43$ proteins decreased markedly in the ISO group compared with the CTL and ISO_DIL groups. These results are in line with previous research (42). Calpains degrade $\mathrm{Cx} 43$ at the protein level, but do not affect the transcription of $\mathrm{Cx} 43$. The mechanism underlying lateralization of connexin following hydrolysis remain to be elucidated, but may be due to myocardial fibrosis, which changes myocardial normal cell morphology and cell spacing, leading to abnormal localization of connexin and ventricular remodeling following ischemia.

Diltiazem, a CCB inhibiting inward $\mathrm{Ca}^{2+}$ flow and reducing intracellular $\mathrm{Ca}^{2+}$ concentration, can effectively expand cardiac blood vessels, increase coronary blood flow and improve myocardial energy metabolism; therefore, it can be widely used in the treatment of cardiovascular diseases (43). Previous research demonstrated that diltiazem can effectively reduce the myocardial cell damage following myocardial hypoxia/ischemia (44), but the majority of evidence is limited to changes in serum markers (45-47). In conclusion, in the present study, chronic administration of isoproterenol induced myocardial fibrosis and was associated with the upregulation of $\mathrm{Ca}^{2+}$. Furthermore, diltiazem improved myocardial remodeling of myocardial ischemia, especially in fibrosis and Cx43. Taken together, the results of the present study suggest that diltiazem serves a protective role in myocardial ischemia. 


\section{References}

1. Pimple P, Shah AJ, Rooks C, Douglas Bremner J, Nye J, Ibeanu I, Raggi $\mathrm{P}$ and Vaccarino V: Angina and mental stress-induced myocardial ischemia. J Psychosom Res 78: 433-437, 2015.

2. Wu H, Ye M, Yang J and Ding J: Endoplasmic reticulum stress-induced apoptosis: A possible role in myocardial ischemia-reperfusion injury. Int J Cardiol 208: 65-66, 2016.

3. Shintani-Ishida K and Yoshida K: Mitochondrial m-calpain opens the mitochondrial permeability transition pore in ischemia-reperfusion. Int J Cardiol 197: 26-32, 2015.

4. Eguchi A, Naito Y, Iwasaku T, Okuhara Y, Morisawa D, Sawada H Nishimura K, Oboshi M, Fujii K, Mano T, et al: Association of dietary iron restriction with left ventricular remodeling after myocardial infarction in mice. Heart Vessels 31: 222-229, 2016.

5. Zhang X, Ambale-Venkatesh B, Bluemke DA, Cowan BR, Finn JP, Kadish AH, Lee DC, Lima JA, Hundley WG, Suinesiaputra A, et al: Information maximizing component analysis of left ventricular remodeling due to myocardial infarction. J Transl Med 13: 343, 2015

6. Travers JG, Kamal FA, Robbins J, Yutzey KE and Blaxall BC: Cardiac fibrosis: The fibroblast awakens. Circ Res 118: 1021-1040, 2016.

7. Hayden MR, Chowdhury N, Govindarajan G, Karuparthi PR, Habibi J and Sowers JR: Myocardial myocyte remodeling and fibrosis in the cardiometabolic syndrome. J Cardiometab Syndr 1: 326-333, 2006.

8. McLenachan JM and Dargie HJ: Ventricular arrhythmias in hypertensive left ventricular hypertrophy. Relationship to coronary artery disease, left ventricular dysfunction, and myocardial fibrosis. Am J Hypertens 3: 735-740, 1990.

9. Jiang F, Liu GS, Dusting GJ and Chan EC: NADPH oxidase-dependent redox signaling in TGF- $\beta$-mediated fibrotic responses. Redox Biol 2: 267-272, 2014.

10. Samarakoon R, Overstreet JM and Higgins PJ: TGF- $\beta$ signaling in tissue fibrosis: Redox controls, target genes and therapeutic opportunities. Cell Signal 25: 264-268, 2013.

11. Tandon A, Tovey JC, Sharma A, Gupta R and Mohan RR: Role of transforming growth factor Beta in corneal function, biology and pathology. Curr Mol Med 10: 565-578, 2010.

12. Kato T, Iwasaki YK and Nattel S: Connexins and atrial fibrillation: Filling in the gaps. Circulation 125: 203-206, 2012.

13. Lambiase PD and Tinker A: Connexins in the heart. Cell Tissue Res 360: 675-684, 2015.

14. Borger MA and Weisel RD: Calcium channel blockers in myocardial and cerebral ischemia: A clinician's review from bench to bedside. Can J Cardiol 15: 333-340, 1999.

15. Ohyama Y, Funao K, Kawabe E, Hayashi D, Yamazaki T, Iga T, Koide D, Ohe K and Kubota K: Calcium channel blockers and myocardial infarction: A case-control study in a Japanese hospital. Pharmacoepidemiol Drug Saf 11: 487-492, 2002.

16. Williams D, Kim KS and Adams-Campbell LL: Survival benefit from calcium channel blockers in elderly blacks following acute myocardial infarction. Ethn Dis 12: 229-233, 2002.

17. Zhang YG, Li YG, Liu BG, Wei RH, Wang DM, Tan XR, $\mathrm{Bu}$ DF, Pang YZ and Tang CS: Urotensin II accelerates cardiac fibrosis and hypertrophy of rats induced by isoproterenol. Acta Pharmacol Sin 28: 36-43, 2007.

18. Hori Y, Kunihiro S, Sato S, Yoshioka K, Hara Y, Kanai K, Hoshi F, Itoh $\mathrm{N}$ and Higuchi S: Doxycycline attenuates isoproterenol-induced myocardial fibrosis and matrix metalloproteinase activity in rats. Biol Pharm Bull 32: 1678-1682, 2009.

19. Ferreira AJ, Santos RA and Almeida AP: Angiotensin-(1-7): Cardioprotective effect in myocardial ischemia/reperfusion. Hypertension 38: 665-668, 2001.

20. Axelsen LN, Calloe K, Braunstein TH, Riemann M, Hofgaard JP, Liang B, Jensen CF, Olsen KB, Bartels ED, Baandrup U, et al: Diet-induced pre-diabetes slows cardiac conductance and promotes arrhythmogenesis. Cardiovasc Diabetol 14: 87, 2015.

21. Kudo-Sakamoto Y, Akazawa H, Ito K, Takano J, Yano M, Yabumoto C, Naito AT, Oka T, Lee JK, Sakata Y, et al: Calpaindependent cleavage of $\mathrm{N}$-cadherin is involved in the progression of post-myocardial infarction remodeling. J Biol Chem 289: 19408-19419, 2014.

22. Livak KJ and Schmittgen TD: Analysis of relative gene expression data using real-time quantitative PCR and the 2(-Delta Delta C(T)) method. Methods 25: 402-408, 2001.

23. Nademanee K, Raju H, de Noronha SV, Papadakis M, Robinson L, Rothery S, Makita N, Kowase S, Boonmee N, Vitayakritsirikul V, et al: Fibrosis, connexin-43, and conduction abnormalities in the brugada syndrome. J Am Coll Cardiol 66: $1976-1986,2015$
24. Garcia Dorado D, Ruiz-Meana M,Inserte J, Rodriguez-Sinovas A and Piper HM: Calcium-mediated cell death during myocardial reperfusion. Cardiovasc Res 94: 168-180, 2012.

25. Bloom S and Davis DL: Calcium as mediator of isoproterenol-induced myocardial necrosis. Am J Pathol 69: 459-470, 1972

26. Monasky MM, Varian KD and Janssen PM: Gender comparison of contractile performance and beta-adrenergic response in isolated rat cardiac trabeculae. J Comp Physiol B 178: 307-313, 2008.

27. Hori Y, Uechi M, Ebisawa T, Yamano S, Yoshioka K and Mutoh K: The influence of gender on cardiac fibrosis induced by sympathetic stimulation. Chin J Physiol 51: 146-151, 2008.

28. Singal PK, Beamish RE and Dhalla NS: Potential oxidative pathways of catecholamines in the formation of lipid peroxides and genesis of heart disease. Adv Exp Med Biol 161: 391-401, 1983

29. Glukhov AV, Balycheva M, Sanchez-Alonso JL, Ilkan Z, Alvarez-Laviada A, Bhogal N, Diakonov I, Schobesberger S, Sikkel MB, Bhargava A, et al: Direct evidence for microdomain-specific localization and remodeling of functional L-type calcium channels in rat and human atrial myocytes. Circulation 132: 2372-2384, 2015.

30. Olwin BB and Storm DR: Calcium binding to complexes of calmodulin and calmodulin binding proteins. Biochemistry 24 : 8081-8086, 1985.

31. Pang $X$ and Sun NL: Calcineurin-NFAT signaling is involved in phenylephrine-induced vascular smooth muscle cell proliferation. Acta Pharmacol Sin 30: 537-544, 2009.

32. Kalyanasundaram A, Lacombe VA, Belevych AE, Brunello L, Carnes CA, Janssen PM, Knollmann BC, Periasamy M and Gyørke S: Up-regulation of sarcoplasmic reticulum $\mathrm{Ca}(2+)$ uptake leads to cardiac hypertrophy, contractile dysfunction and early mortality in mice deficient in CASQ2. Cardiovasc Res 98: 297-306, 2013

33. WeiHY, GuoZQ and Cui SJ: Apocalmodulin and $\mathrm{Ca}^{2+}$-independent calmodulin-binding proteins. Prog Biochem Biophys 34: 124-131, 2007 (In Chinese)

34. Wei LH, Huang XR, Zhang Y, Li YQ, Chen HY, Heuchel R, Yan BP, Yu CM and Lan HY: Deficiency of Smad7 enhances cardiac remodeling induced by angiotensin II infusion in a mouse model of hypertension. PLoS One 8: e70195, 2013

35. Kessler Icekson G, Schlesinger H, Freimann S and Kessler E Expression of procollagen C-proteinase enhancer-1 in the remodeling rat heart is stimulated by aldosterone. Int $\mathrm{J}$ Biochem Cell Biol 38: 358-365, 2006.

36. Saffitz JE: Douglas P. Zipes Lecture. Biology and pathobiology of cardiac connexins: From cell to bedside. Heart Rhythm 3: 102-107, 2006.

37. Söhl G and Willecke K: Gap junctions and the connexin protein family. Cardiovasc Res 62: 228-232, 2004.

38. Severs NJ, Bruce AF, Dupont E and Rothery S: Remodelling of gap junctions and connexin expression in diseased myocardium. Cardiovasc Res 80: 9-19, 2008.

39. Halidi N, Alonso F, Burt JM, Bény JL, Haefliger JA and Meister JJ: Intercellular calcium waves in primary cultured rat mesenteric smooth muscle cells are mediated by connexin 43. Cell Commun Adhes 19: 25-37, 2012.

40. Lurtz MM and Louis CF: Intracellular calcium regulation of connexin43. Am J Physiol Cell Physiol 293: C1806-C1813, 2007.

41. Goll DE, Thompson VF, Li H, Wei W and Cong J: The calpain system. Physiol Rev 83: 731-801, 2003.

42. Zhang W, Ma X, Zhong M, Zheng Z, Li L, Wang Z and Zhang Y: Role of the Calpain system in pulmonary vein connexin remodeling in dogs with atrial fibrillation. Cardiology 112: 22-30, 2009.

43. Anderson ME: Calmodulin kinase and L-type calcium channels; a recipe for arrhythmias? Trends Cardiovasc Med 14: 152-161, 2004.

44. Kröner A, Seitelberger R, Schirnhofer J, Bernecker O, Mallinger R, Hallström S, Ploner M and Podesser BK: Diltiazem during reperfusion preserves high energy phosphates by protection of mitochondrial integrity. Eur J Cardiothorac Surg 21: 224-231, 2002.

45. Hegde A, Amaria T, Mandke A and Mandke NV: Comparative study of perioperative infusion of diltiazem and nicorandil on myocardial protection during OPCAB surgery. Ann Card Anaesth 8: 49-54, 2005.

46. Chouairi S, Carrie D and Puel J: Myocardial protection with calcium-channel blockers during ischaemia and reperfusion by PTCA, Eur Heart J 16 (Suppl H): S3-S8, 1995.

47. Rousseau G, Provost P and Latour JG: Sustained myocardial protection by clentiazem (TA-3090) after a 90-minute coronary occlusion and 72 hours of reperfusion in dogs with collateral flow. J Cardiovasc Pharmacol 22: 264-272, 1993. 